\title{
Celebrating the Practice of Distance Learning
}

\author{
Anthony A. Piña ${ }^{1} \cdot$ Linda L. Campion ${ }^{2}$
}

Published online: 19 August 2017

(C) Association for Educational Communications \& Technology 2017

The recent announcement of Purdue University's acquisition of Kaplan University has brought the conversation of online and distance learning to the forefront of conversations in higher education. It is well-documented that online education has been the fastest growing sector of college and university enrollments throughout the new millennium. While MOOCs have dominated much of the popular literature in recent years, the real story may be that in a time of postsecondary enrollment declines, online and distance learning have continued to grow, with new online programs, from certificates to doctorates, springing up at colleges and universities across the globe.

It appears to be an opportune time for us, along with our colleagues from AECT's Division of Distance Learning (DDL), to celebrate the practice of distance learning. We wish to extend our thanks to Dr. Charles Hodges for allowing us to edit this special issue of Tech Trends and for the support of our colleagues on the Board of the Division of Distance Learning (AECT-DLL): Tonia Dousay, Cindy York, Florence Martin, Ayesha Sadaf, Larisa Olesova, Yu-Hui Ching, Michele Estes and Victoria Lowell. We are particularly grateful to Phillip Harris and Larry Vernon from AECT Headquarters for the promotion of this issue and its call for manuscripts.

We were surprised at the level of interest by our colleagues for this special issue. Almost immediately after the issue was

Anthony A. Piña

APina@ sullivan.edu

Linda L. Campion

CAMPIONL16@ecu.edu

Sullivan University, Louisville, KY, USA

2 East Carolina University, Greenville, NC, USA publicized at the 2016 AECT Annual Convention, proposals and manuscripts starting arriving. The selection of ten articles from a pool of 54-a 19\% acceptance rate-was extremely difficult, given the large number of high quality and interesting proposals. We are grateful for our colleagues who served as peer reviewers for this issue.

Our vision for this Special Issue was to provide Tech Trends readers with a variety of articles that include practical and applicable ideas, strategies, and techniques on topics related to distance learning. Do you want to know what students are saying about online courses/learning and how their online instructors could help them learn more successfully? Then you'll want to dive into the Watson, Bishop and FerdinandJames article for a full explanation based on a dissertation study. The instructional strategies preferred by the participants are explored and boiled down into The Top 10.

Sound instructional practices are key to designing learning in the online learning environment. Christopher Rozitis discusses instructional design competencies needed for effective modification of learning objects within a high school online course environment. The resulting competencies from this Delphi study are presented.

Learning design meetings - how do we make them more successful? Denise Shaver maps a process used at her institution in order to "set the tone for success and reduce faculty resistance to the process of online course development."

Faculty development for online teaching is not a one-size fits all practice. Jason Rhode, Stephanie Richter and Tracy Miller are presenting on their research based on a selfassessment instrument which drives the design and crafting of their workshops, immersive online experiences, consultations and just-in-time resources used to support their faculty in this critical developmental process taking individual experience and skill into account. 
Video can be a powerful instructional tool and Jenny Wakefield, Terry DiPaolo and Leila Mills provide a primer for those new to producing video for online courses. They propose a detailed four-step model of planning, development, delivery and reflection to be used for integrating video into online courses.

Is it possible to improve our social presence through the use of asynchronous video feedback and individual communications with our online students? Rick West, Jason Jay, Matt Armstrong and Jered Borup present strategies to 1) improve efficiency, 2) increase engagement in teaching and learning and 3) increase effectiveness in teaching and learning through this practice. These strategies are detailed and easily adopted in our profession.

Xeturah Woodley, Cecilia Hernandez, Julia Parra and Beyan Negash establish the importance of recognizing and acting upon the increasingly diverse makeup of our distance learners. They provide several highly practical activities that can be integrated into online courses, serving as best practices for culturally responsive teaching.
Michael Kung takes the conversation about student diversity and cultural awareness into a global realm by focusing upon recommendations for faculty, instructional designers and international students that can help to increase the success for international students enrolled in online and hybrid courses at U.S. institutions.

Michael Matthews, Gregory Williams, Jason McDonald, and Stephen Yanchar turn the focus to the instructional designer and the role that empathy plays on the practice of online instructional design, including tensions between common design practices and designer empathy.

Finally, Dale Crowe, Martin LaPierre and Mansureh Kebritchi look to the future of knowledge-based systems. Distinguishing between artificial intelligence and augmented intelligence, Crowe and his colleagues outline the possibility of utilizing advanced knowledge-based systems, including IBM's Watson, to build applications to enhance and extend instructional design and distance learning.

We hope that you find this special DDL issue to be enlightening and instructive. 